\title{
ON THE AREAS OF MIDPOINT POLYGONS
}

\author{
CHAO WANG AND ZHONGZI WANG
}

Abstract. For a polygon $V_{1} \ldots V_{n}$ in the Euclidean plane, let $V_{1}^{1} \ldots V_{n}^{1}$ denote its midpoint polygon. By induction, its $m$-th midpoint polygon $V_{1}^{m} \ldots V_{n}^{m}$ is defined to be the midpoint polygon of $V_{1}^{m-1} \ldots V_{n}^{m-1}$. In this paper, we will give different kinds of formulas of the area of $V_{1}^{m} \ldots V_{n}^{m}$. We will describe the limit behavior of the area as $m$ goes to infinity, and we will determine the infimum and the supremum of the area among all convex $V_{1} \ldots V_{n}$ with a fixed area. Some affine invariants derived from the area will also be discussed.

Mathematics subject classification (2010): 51M25, 52A38, 52A40.

Keywords and phrases: Area, midpoint polygon, affine invariant.

\section{REFERENCES}

[1] www.techhouse.org/ ${ }^{m d p / m i d p o i n t / i n d e x . p h p ~}$

[2] E. Berlekamp, E. Gilbert, F. Sinden, A polygon problem, Amer. Math. Monthly 72 (1965), 233-241.

[3] F. Bachmann, E. Schmidt, $n$-gons, Translated from the German by Cyril W. L. Garner. Mathematical Expositions, No. 18. University of Toronto Press, Toronto, Ont.-Buffalo, N. Y., 1975.

[4] G. ChANG, P. DAVIS, Iterative processes in elementary geometry, Amer. Math. Monthly 90 (1983), no. 7, 421-431.

[5] H. Croft, K. Falconer, R. GuY, B25. Sequences of polygons and polyhedra, Unsolved Problems in Geometry, Springer, (1991), pp. 76-78.

[6] G. Chang, T. Sederberg, Over and over again, New Mathematical Library, 39. Mathematical Association of America, Washington, DC, 1997.

[7] A. N. Elmachtoub, C. F. VAn LoAn, From random polygon to ellipse: an eigenanalysis, SIAM Rev. 52 (2010), no. 1, 151-170.

[8] R. J. GARDNER, Geometric tomography, Encyclopedia of Mathematics and its Applications, 58 (2nd ed.), Cambridge University Press (2006).

[9] F. Gomez-Martin, P. Taslakian, G. Toussaint, Convergence of the shadow sequence of inscribed polygons, http://oa.upm.es/4442/, (2008).

[10] D. Ismailescu, M. Kim, K. Lee, S. Lee, T. Park, Area problems involving Kasner polygons, arXiv:0910.0452 math.MG (CO).

[11] E. KASNER, The group generated by central symmetries, with application to polygons, The American Mathematical Monthly (1903), 10(3), 57-63.

[12] Z. Z. WANG, C. WANG, On the area of midpoint pentagon, Mathematics Bulletin (Chinese) (2016), no. $11,54-58$.

[13] C. WANG, Z. Z. WANG, The limit shapes of midpoint polygons in $\mathbb{R}^{3}$, J. Knot Theory Ramifications 28 (2019), no. 10, 1950062, 17 pp.

[14] R. M. ZвiEK, The Pentagon Problem: Geometric Reasoning with Technology, The Mathematics Teacher 89 (February 1996) 86-90. 